\title{
artigo
}

\section{Os principais desafios dos enfermeiros na liderança em enfermagem}

\author{
The key challenges facing nurses in nursing leadership \\ Los principales desafíos para las enfermeras en el liderazgo de enfermería
}

\begin{abstract}
RESUMO
Objetivo: 0 presente estudo trata-se de uma revisão integrativa que tem como objetivo identificar o que a literatura científica tem abordado acerca dos principais desafios enfrentados pelos enfermeiros na liderança em enfermagem. Método: Os estudos utilizados nesta revisão foram coletados por meio das bases de dados PubMed, Scielo e Cochrane. 0 critério de inclusão foi o ano de publicação e o critério de exclusão foi a ausência das palavras-chave no título e no resumo. Resultado: As buscas resultaram em 1.430 artigos, dos quais 24 atenderam aos critérios de elegibilidade e foram incluídos nesta revisão. Conclusão: A partir da leitura e análise dos artigos, foram identificados 12 principais desafios, sendo que o desenvolvimento de habilidades de liderança foi o principal tema abordado. Devido a complexidade de compreender e superar os desafios identificados, sugere-se novos estudos sobre liderança em enfermagem.
\end{abstract}

DESCRITORES: Enfermeiro; Liderança; Administração.

\section{ABSTRACT}

Objective: This study is an integrative review that aims to identify what the scientific literature has addressed the key challenges in nursing leadership. Method: The studies used in this article were collected on the PubMed, Scielo, and Cochrane databases. The inclusion criterion was the year of publication and the exclusion criterion was the absence of keywords in the title and abstract. Result: The searches resulted in 1,430 articles, 24 met the eligibility criteria and were included in this review. Conclusion: After reading and analyzing the articles, 12 main challenges were identified, with developing leadership skills being the main topic addressed. Due to the complexity of understanding and overcoming the challenges identified, new studies on nursing leadership are suggested.

DESCRIPTORS: Nurse; Leadership; Administration.

\section{RESUMEN}

Objetivo: El presente estudio es una revisión integradora que tiene como objetivo identificar lo que la literatura científica ha abordado sobre los principales desafíos que enfrentan las enfermeras en el liderazgo de enfermería. Método: Los estudios utilizados en esta revisión se recopilaron mediante las bases de datos PubMed, Scielo y Cochrane. El criterio de inclusión fue el año de publicación y el criterio de exclusión fue la ausencia de palabras clave en el título y el resumen. Resultado: Las búsquedas dieron como resultado 1430 artículos, 24 cumplieron los criterios de elegibilidad y se incluyeron en esta revisión. Conclusión: A partir de la lectura y análisis de los artículos se identificaron 12 desafíos principales, siendo el desarrollo de habilidades de liderazgo el tema principal abordado. Debido a la complejidad de comprender y superar los desafíos identificados, se sugieren nuevos estudios sobre liderazgo en enfermería.

DESCRIPTORES: Enfermera; Liderazgo; Administración.

RECEBIDO EM: 10/03/2021 APROVADO EM: 22/03/2021

\section{Aryadne O'neil de Oliveira Souza Lourenço}

Enfermeira Referência do Setor de Endoscopia. Enfermeira com Pós-graduação em Gestão e Liderança em Enfermagem e Formação em Coaching Integrado. Santa Casa de Misericórdia de Barretos

ORCID: 0000-0003-0236-1494

\section{Luciene Almeida da Silva}

Enfermeira Médio Gerente do Setor de Pronto Atendimento. Enfermeira com Pós-graduação em Gestão e Liderança em Enfermagem. Santa Casa de Misericórdia de Barretos.

ORCID: 0000-0001-5307-6875 


\section{Maria Aurélia da Silveira Assoni}

Enfermeira Coordenadora da Educação Corporativa. Enfermeira Mestre em Ensino em Saúde e MBA Executivo em Saúde. Hospital de Amor

ORCID: 0000-0002-6460-9267

\section{INTRODUÇ̃̃O}

C onstantemente ocorrem mudanças nos cenários político, econômico e social, que fazem com que o mercado de trabalho tenha que se adaptar aos novos moldes, exigindo cada vez mais novas competências dos profissionais. Dentre estas, a prática da liderança tem sido amplamente requerida em diversos ambientes de trabalho. ${ }^{1}$

No Brasil, o exercício da profissão de enfermagem é regulamentado pelo Conselho Federal de Enfermagem (COFEN) por meio da Lei ${ }^{\circ} 7.498 / 86$. O Artigo 11 desta Lei, descreve as atividades atribuídas ao enfermeiro. Grande parte destas atividades estão relacionadas ao planejamento, organização, coordenação, execução e avaliação dos serviços da assistência de enfermagem, o que demonstra que o trabalho do enfermeiro excede ao cuidado e a assistência. ${ }^{2}$

Não é por acaso que a maior parte dos cargos de gestão hospitalar é ocupada por enfermeiros, visto que este profissional já é direcionado para a liderança em sua formação. Os enfermeiros desenvolvem um papel importante enquanto gestores nos cuidados de saúde prestados à população, tendo em vista sua competência em liderar e lidar com diversas situações que requerem análise e compreensão de todo o processo de trabalho, assim como, constante tomada de decisões. ${ }^{3}$

Neste contexto, o objetivo desse estudo é identificar o que a literatura científica tem abordado sobre os principais desafios enfrentados pelos enfermeiros acerca da liderança e discutir essas informações em forma de revisão integrativa com o intuito de apoiar o desenvolvimento profissional dos enfermeiros em relação à prática da liderança em enfermagem.

\section{MÉTODOS}

O presente estudo trata-se de uma re-
Constantemente

ocorrem mudanças

nos cenários político,

econômico e social,

que fazem com

que o mercado de

trabalho tenha que

se adaptar aos novos

moldes, exigindo

cada vez mais novas

competências dos

profissionais. Dentre

estas, a prática

da liderança tem

sido amplamente

requerida em

diversos ambientes

de trabalho. visão integrativa da literatura, uma metodologia que tem como finalidade agrupar e integrar estudos referentes a um determinado assunto. A revisão integrativa proporciona a síntese das informações e a incorporação de resultados de estudos significativos na prática, sendo muito utilizada na área da saúde. ${ }^{4}$

O material utilizado foi coletado na literatura científica com o auxílio dos Descritores em Ciências da Saúde (DeCS), Medical Subject Headings (MeSH) e das bases de dados PubMed, Scielo e Cochrane.

Para a construção da estratégia de busca, as palavras-chave enfermeiro, liderança e administração foram inseridas no DeCs, resultando nos descritores nurse, leadership e administration. Posteriormente, os descritores foram inseridos no $\mathrm{MeSH}$, com o intuito de encontrar todos os seus sinônimos indexados, o que permitiu a elaboração da seguinte estratégia de busca: (Nurse[Title] OR Nurses[Title]) AND (Leadership[Title] OR Influentials[Title] OR Administration[Title]).

$\mathrm{O}$ critério de inclusão utilizado foi o ano de publicação, sendo selecionados apenas os artigos publicados nos últimos 5 anos. O critério de exclusão, por sua vez, foi a ausência das palavras-chave no título e no resumo.

Para selecionar os estudos que se enquadravam ao tema e ao propósito desta revisão, foram lidos os títulos e resumos dos artigos científicos publicados nos últimos 5 anos, buscando as seguintes palavras-chave: desafios, enfermeiros, liderança e administração. Artigos em português, inglês e espanhol foram considerados neste estudo.

Após a seleção dos artigos, foi realizada uma leitura mais aprofundada, com o intuito de identificar o que os estudos abordaram acerca dos principais desafios dos enfermeiros na liderança em enfermagem. Os dados foram analisados e tratados com o auxílio de ferramentas do Pacote Office (Word e Excel). 


\section{artigo}

Lourenço, A.O.O.S.; Almeida da Silva, L.; Assoni, M.A.S.

Os principais desafios dos enfermeiros na liderança em enfermagem

Figura 1. Quantidade de artigos encontrados, elegíveis e incluídos na revisão integrativa.
Critério de exclusão:

Ausência das palavras-chave no título e no resumo

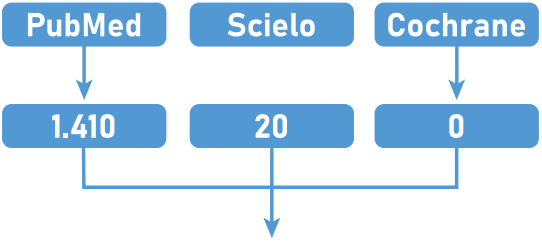

\subsection{0}

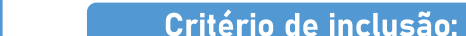
Publicado nos últimos 5 anos

\section{0}

Elegiveis

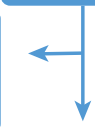

24

Incluidos

\section{RESULTADOS}

Foram encontrados 1.430 artigos, sendo que 24 atenderam aos critérios de inclusão e exclusão e foram incluídos nesta revisão (Figura 1). O quadro 1 apresenta a caracterização dos estudos incluídos na revisão integrativa mediante base de dados, título, tipo de estudo, autores, periódico e ano de publicação.

A Figura 2 apresenta os principais desafios identificados a partir da leitura e análise dos artigos. Os desafios foram subdivididos de acordo com o número de vezes que o tema foi abordado. Pode-se observar que "desenvolver habilidades de liderança" foi o principal tema abordado, estando presente em 6 dos 24 artigos selecionados.

\section{DISCUSSÃO}

O desenvolvimento de habilidades de

Quadro 1. Apresentação da caracterização dos estudos incluídos na revisão integrativa mediante: Base de dados, Título, Tipo de estudo, Autores, Periódico e ano de publicação.

\begin{tabular}{|c|c|c|c|c|}
\hline $\begin{array}{l}\text { BASE DE } \\
\text { DADOS }\end{array}$ & TítULO & TIPO DE ESTUDO & AUTORES & $\begin{array}{l}\text { PERIÓDICO E ANO DE } \\
\text { PUBLICAÇÃO }\end{array}$ \\
\hline PubMed & $\begin{array}{c}\text { Growing Nurse Leaders: Their Perspec- } \\
\text { tives on Nursing Leadership and Today's } \\
\text { Practice Environment }\end{array}$ & Estudo qualitativo & $\begin{array}{c}\text { Dyess S, Sherman R, Pratt } \\
\text { B, Chiang-Hanisko L }\end{array}$ & $\begin{array}{l}\text { OJIN: The Online Journal } \\
\text { of Issues in Nursing, } \\
2016\end{array}$ \\
\hline PubMed & $\begin{array}{c}\text { Nurse Practitioner Autonomy and } \\
\text { Relationships with Leadership Affect } \\
\text { Teamwork in Primary Care Practices: A } \\
\text { Cross-Sectional Survey }\end{array}$ & $\begin{array}{l}\text { Pesquisa } \\
\text { transversal }\end{array}$ & Poghosyan L, Liu J & $\begin{array}{l}\text { Journal of General } \\
\text { Internal Medicine, } 2016\end{array}$ \\
\hline Scielo & $\begin{array}{l}\text { Professional competences and organiza- } \\
\text { tional strategies of nursing managers }\end{array}$ & Estudo qualitativo & $\begin{array}{l}\text { Camelo SHH, Rocha FLR, } \\
\text { Chaves LDP, Silva VLS, } \\
\text { Soares MI }\end{array}$ & $\begin{array}{l}\text { Ciencia y Enfermeria, } \\
2016\end{array}$ \\
\hline PubMed & $\begin{array}{l}\text { Contributions of Paulo Freire to unders- } \\
\text { tanding the dialogic leadership exercise of } \\
\text { nurses in the hospital setting }\end{array}$ & Estudo qualitativo & $\begin{array}{l}\text { Amestoy SC, Oliveira AFL, } \\
\text { Thofehrn MB, Trindade LL, } \\
\text { Santos BP, Bao ACP }\end{array}$ & $\begin{array}{l}\text { Revista Gaúcha de En- } \\
\text { fermagem, } 2017\end{array}$ \\
\hline PubMed & $\begin{array}{c}\text { Toward a mediation model for nurses' } \\
\text { well-being and psychological distress } \\
\text { effects of quality of leadership and social } \\
\text { support at work }\end{array}$ & Pesquisa transversal & $\begin{array}{c}\text { Van der Heijden BIJM, } \\
\text { Mulder RH, König C, Ansel- } \\
\text { mann V }\end{array}$ & Medicine, 2017 \\
\hline Scielo & $\begin{array}{l}\text { Leadership in nursing: Challenges and } \\
\text { possibilities }\end{array}$ & Editorial & Balsanelli AP & $\begin{array}{l}\text { Acta Paulista de Enfer- } \\
\text { magem, } 2017\end{array}$ \\
\hline PubMed & $\begin{array}{l}\text { Nurse Managers' Emotional Intelligence } \\
\text { and Effective Leadership: A Review of the } \\
\text { Current Evidence }\end{array}$ & Revisão de literatura & Prezerakos PE & $\begin{array}{l}\text { The Open Nursing Jour- } \\
\text { nal, } 2018\end{array}$ \\
\hline PubMed & $\begin{array}{c}\text { Nurse Managers' Emotional Intelligence } \\
\text { and Effective Leadership: A Review of } \\
\text { Current Evidence }\end{array}$ & Artigo comentário & Al-Motlaq M & $\begin{array}{l}\text { The Open Nursing Jour- } \\
\text { nal, } 2018\end{array}$ \\
\hline
\end{tabular}




\begin{tabular}{|c|c|c|c|c|}
\hline PubMed & $\begin{array}{l}\text { Exploring the role of advanced nurse } \\
\text { practitioners in leadership }\end{array}$ & Estudo qualitativo & Anderson C & Nursing Standard, 2018 \\
\hline PubMed & $\begin{array}{l}\text { Health promoting leadership: A quali- } \\
\text { tative study from experienced nurses' } \\
\text { perspective }\end{array}$ & Estudo qualitativo & $\begin{array}{l}\text { Furunes T, Kaltveit A, } \\
\text { Akerjordet K }\end{array}$ & $\begin{array}{l}\text { Journal of Clinical Nursing, } \\
2018\end{array}$ \\
\hline PubMed & $\begin{array}{l}\text { Rationing nurses: Realities, practicali- } \\
\text { ties, and nursing leadership theories }\end{array}$ & Investigação & Fast O, Rankin J & Nursing Inquiry, 2018 \\
\hline Scielo & $\begin{array}{l}\text { Management challenges for best } \\
\text { practices of the Kangaroo Method in } \\
\text { the Neonatal ICU }\end{array}$ & Estudo qualitativo & $\begin{array}{l}\text { Silva LJ, Leite JL, Silva } \\
\text { TP, Silva IR, Mourão PP, } \\
\text { Gomes TM }\end{array}$ & $\begin{array}{l}\text { Revista Brasileira de Enfer- } \\
\text { magem, } 2018\end{array}$ \\
\hline PubMed & $\begin{array}{l}\text { Barriers to nurse leadership in an } \\
\text { Indonesian hospital setting }\end{array}$ & Estudo qualitativo & Wardani E, Ryan T & $\begin{array}{l}\text { Journal of Nursing Mana- } \\
\text { gement, } 2019\end{array}$ \\
\hline PubMed & $\begin{array}{l}\text { Preparing Nurse Managers for Au- } \\
\text { thentic Leadership }\end{array}$ & Estudo transversal & Frasier N & $\begin{array}{l}\text { The Journal of Nursing } \\
\text { Administration, } 2019\end{array}$ \\
\hline PubMed & $\begin{array}{c}\text { An inductive qualitative approach to } \\
\text { explore Nurse Practitioners views on } \\
\text { leadership and research: An internatio- } \\
\text { nal perspective }\end{array}$ & Estudo qualitativo & $\begin{array}{l}\text { Ryder M, Jacob E, Hen- } \\
\text { dricks J }\end{array}$ & $\begin{array}{l}\text { Journal of Clinical Nursing, } \\
2019\end{array}$ \\
\hline PubMed & $\begin{array}{l}\text { Developing effective nurse leadership } \\
\text { skills }\end{array}$ & Estudo qualitativo & Major D & Nursing Standard, 2019 \\
\hline PubMed & $\begin{array}{l}\text { The Effect of Leadership Interventions } \\
\text { on Staff Nurse Job Enjoyment and } \\
\text { Leadership Perception }\end{array}$ & Estudo qualitativo & Correa PB, Bacon CT & $\begin{array}{l}\text { The Journal of Nursing } \\
\text { Administration, } 2019\end{array}$ \\
\hline Scielo & $\begin{array}{l}\text { Being an entrepreneur in nursing: } \\
\text { challenges to nurses in a strategic } \\
\text { leadership position }\end{array}$ & $\begin{array}{l}\text { Estudo qualitativo, des- } \\
\text { critivo e exploratório }\end{array}$ & $\begin{array}{l}\text { Richter SA, Santos EP, } \\
\text { Kaiser DE, Capellari C, } \\
\text { Ferreira GE }\end{array}$ & $\begin{array}{l}\text { Acta Paulista de Enferma- } \\
\text { gem, } 2019\end{array}$ \\
\hline PubMed & $\begin{array}{l}\text { Influence of nurse and midwife } \\
\text { managerial leadership styles on job } \\
\text { satisfaction, intention to stay, and } \\
\text { services provision in selected hospitals } \\
\text { of Rwanda }\end{array}$ & $\begin{array}{l}\text { Estudo transversal } \\
\text { descritivo }\end{array}$ & $\begin{array}{l}\text { Ngabonzima A, Asingi- } \\
\text { zwe D, Kouveliotis K }\end{array}$ & BMC Nursing, 2020 \\
\hline PubMed & $\begin{array}{c}\text { Transformational leadership: journal } \\
\text { club for emergency and intensive care } \\
\text { nurse managers }\end{array}$ & Pesquisa qualitativa & $\begin{array}{l}\text { Pereira MV, Spiri WC, } \\
\text { Spagnuolo RS, Juliani } \\
\text { CMCM. }\end{array}$ & $\begin{array}{c}\text { Revista Brasileira de Enfer- } \\
\text { magem, } 2020\end{array}$ \\
\hline PubMed & $\begin{array}{l}\text { 'It's the relationship you develop with } \\
\text { them': emotional intelligence in nurse } \\
\text { leadership. A qualitative study }\end{array}$ & Estudo qualitativo & Mansel B, Einion A & $\begin{array}{l}\text { British Journal of Nursing, } \\
2020\end{array}$ \\
\hline PubMed & $\begin{array}{c}\text { Clinical Nurses' Perceptions of Authen- } \\
\text { tic Nurse Leadership and Healthy Work } \\
\text { Environment }\end{array}$ & $\begin{array}{l}\text { Estudo descritivo, cor- } \\
\text { relacional e transversal }\end{array}$ & $\begin{array}{l}\text { Raso R, Fitzpatrick JJ, } \\
\text { Masick K }\end{array}$ & $\begin{array}{l}\text { The Journal of Nursing } \\
\text { Administration, } 2020\end{array}$ \\
\hline Scielo & $\begin{array}{c}\text { Leadership in the perspective of Family } \\
\text { Health Strategy nurses }\end{array}$ & Pesquisa-ação & $\begin{array}{c}\text { Oliveira C, Santos LC, } \\
\text { Andrade J, Domingos TS, } \\
\text { Spiri WC } \\
\end{array}$ & $\begin{array}{l}\text { Revista Gaúcha de Enfer- } \\
\text { magem, } 2020\end{array}$ \\
\hline Scielo & $\begin{array}{l}\text { Transformational leadership in nursing } \\
\text { practice: challenges and strategies }\end{array}$ & $\begin{array}{l}\text { Estudo qualitativo e } \\
\text { exploratório }\end{array}$ & $\begin{array}{c}\text { Ferreira VB, Amestoy } \\
\text { SC, Silva GTR, Trindade } \\
\text { LL, Santos IAR, Varanda } \\
\text { PAG }\end{array}$ & $\begin{array}{c}\text { Revista Brasileira de Enfer- } \\
\text { magem, } 2020\end{array}$ \\
\hline
\end{tabular}

liderança foi o principal desafio identificado. Os estudos abordaram as barreiras que dificultam o desenvolvimento das habilidades de liderança, ${ }^{5,6,7}$ o desenvolvimento da liderança em enfermeiros recém-qualificados, 8 fatores relacionados à equipe, aos processos de liderança e ao líder ${ }^{9} \mathrm{e}$ os desafios e estratégias adotados para o exercício da liderança. ${ }^{10}$

Os principais fatores mencionados como barreiras ao desenvolvimento das habilidades de liderança foram: pers- 
Figura 2. Os principais desafios dos enfermeiros na liderança em enfermagem identificados na literatura científica.

\section{DESAFIOS IDENTIFICADOS}

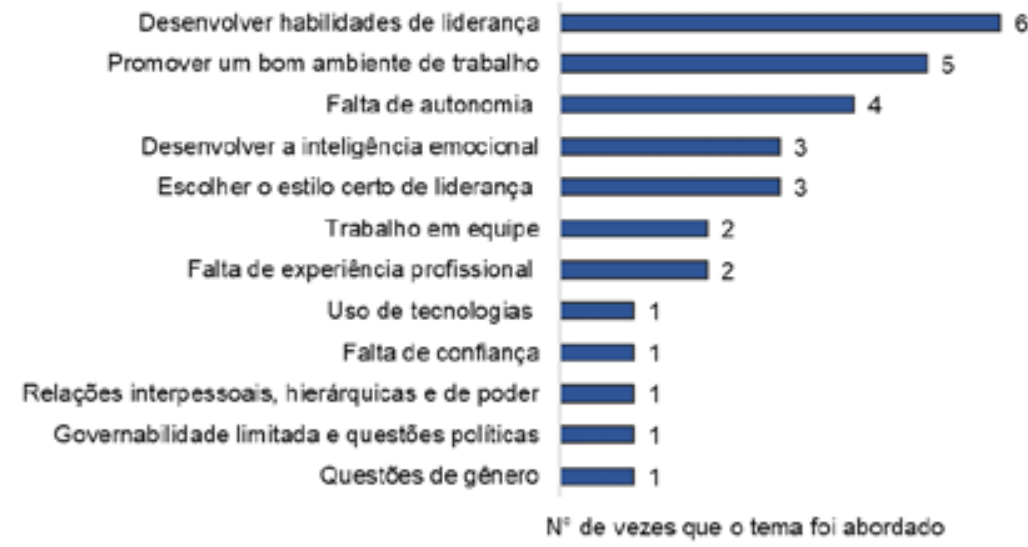

pectivas restritas, ${ }^{5}$ questões de gênero, ${ }^{5,7}$ barreiras contratuais e profissionais, ${ }^{5,7}$ questões hierárquicas e de estrutura organizacional, ${ }^{5,10}$ ritmo acelerado e mudanças repentinas no ambiente de trabalho, ${ }^{6}$ falta de apoio por parte da instituiçãa $0^{7,8,10} \mathrm{e}$ inexperiência profissional e jovialidade. ${ }^{8,10}$

Os estudos também apresentaram caminhos para superar este desafio. Wardani,Ryan5 relataram que, é necessário apoio e educação persistentes. Para Major, ${ }^{8}$ enfermeiros seniores e organizações de saúde não devem esperar que enfermeiros recém-qualificados desenvolvam habilidades de liderança sem apoio, portanto, caminhos educacionais claros são necessários ao longo do treinamento. Anderson ${ }^{7}$ alertou ainda que, se este apoio não estiver disponível, o envelhecimento da população resultará em uma redução significativa de líderes de enfermagem disponíveis no futuro.

Promover um bom ambiente de trabalho foi segundo tema mais abordado. Os estudos apontaram que nem sempre as relações entre enfermeiros-líderes e equipe favorecem um ambiente de trabalho saudável e estimulante, ${ }^{11}$ o que torna o gerenciamento de conflitos e a busca por um bom ambiente de trabalho, grandes desafios para os enfermeiros. ${ }^{12}$

O estudo realizado por Van der Heijden et al..$^{13}$ investigou os efeitos da qualidade da liderança e do apoio social no trabalho sobre o bem-estar e sofrimento psíquico dos enfermeiros. Os autores relataram que as influências positivas e negativas relacionadas à qualidade da liderança, ao apoio social do líder e dos colegas de trabalho e ao próprio ambiente de trabalho, tem grande influência sobre o bem-estar e sofrimento psíquico dos enfermeiros.

Farunes et al. ${ }^{14}$ e Raso et al. ${ }^{15}$ também contemplaram a percepção dos enfermeiros sobre o ambiente de trabalho e apontaram a importância de se ter um ambiente promotor da saúde e que busque constantemente por melhorias. Para Farunes et al., ${ }^{14}$ ambientes de trabalho promotores de saúde permitem que os enfermeiros floresçam e ter ampla autonomia é importante para que os enfermeiros enxerguem os desafios como oportunidades para desenvolver suas competências.

A questão da autonomia mencionada anteriormente por Farunes et al., ${ }^{14}$ foi abordada como principal desafio em outros estudos ${ }^{16,17,18}$ e apresentou relação com trabalho em equipe e questões de gênero. Além disso, fatores como governabilidade limitada e questões políticas, também apresentaram influência sobre a autonomia na tomada de decisões. ${ }^{19}$

Os estudos de Poghosyan,Liu ${ }^{16}$ e Silva et al. ${ }^{17}$ demonstraram que autonomia e relações favoráveis com a liderança melhoram o trabalho em equipe. Contudo, o estudo de Silva et al. ${ }^{17}$ apontou que o maior desafio da liderança é ter autonomia para a tomada de decisões e que a falta de autonomia é um dos fatores que dificultam o estabelecimento de laços de confiança e, consequentemente, o trabalho em equipe, que, por sua vez, está diretamente vinculado ao desenvolvimento pessoal e profissional dos indivíduos e à qualidade do atendimento ao paciente.

Além da relação com o trabalho em equipe, é possível observar no estudo de Richter et al., ${ }^{18}$ realizado com o intuito de conhecer os desafios de enfermeiras em posição estratégica de liderança ao desenvolvimento de ações empreendedoras, uma relação entre falta de autonomia e questão de gênero. Segundo os autores, ao mesmo tempo em que é requerido alto 
desempenho institucional destas mulheres que ocupam posições estratégicas de liderança, as mesmas dispõem de pouca autonomia e credibilidade, o que as imobilizam no desenvolvimento de ações empreendedoras.

O desenvolvimento da inteligência emocional também foi um tema apontado como desafio para os enfermeiros. Prezerakos $^{20}$ avaliou a relação entre a inteligência emocional dos gerentes de enfermagem e a liderança eficaz. Os resultados mostraram que a inteligência emocional é uma ferramenta importante para o alcance de uma gestão eficaz, porém seu desenvolvimento ainda é um grande desafio para os enfermeiros. Este estudo feito por Prezerakos $^{20}$ foi comentado e reforçado por Al-Motlaq. ${ }^{21}$

Mansel, Einion ${ }^{22}$ também estudaram a inteligência emocional e sua relação com a liderança em enfermagem. Os autores relataram que os desafios para o desenvolvimento emocional identificados pelos líderes foram em relação ao tempo, pressão e níveis insuficientes de pessoal. Estes fatores parecem suprimir o potencial de se tornarem líderes mais eficazes usando a inteligência emocional. Os autores sugerem alocar tempo e recursos suficientes para o engajamento da equipe em atividades que possam aumentar sua capacidade de desenvolver e aplicar a inteligência emocional em sua vida profissional.

Escolher o estilo certo de liderança foi apontado como um desafio que influencia diretamente a relação entre os líderes e sua equipe. Frasier ${ }^{23}$ estudou o uso da liderança autêntica pelos gerentes de enfermagem e relatou que os gerentes perceberam uma maior autoconsciência e os subordinados perceberam mudanças positivas no comportamento dos gerentes. O estudo feito por Ngabonzima et al., ${ }^{24}$ para avaliar a relação entre os estilos de liderança e a satisfação no trabalho, também mostrou que o nível de satisfação variou de acordo com o estilo de liderança adotado. Balsanelli ${ }^{25}$ sugeriu ainda, a utilização de instrumentos de medida, que podem otimizar o mapeamento dos estilos de liderança dos enfermeiros e adotar planos de desenvol- vimento individual, com metas a serem atingidas, estimulando assim, o desenvolvimento desta competência.

\section{A literatura}

apresentou dados

e informações

\section{relevantes que}

permitiram entender

quais são os

\section{principais desafios}

dos enfermeiros

na liderança em

enfermagem. Foram

identificados 12

desafios, sendo

que, "desenvolver

habilidades de

liderança" foi o

principal desafio

abordado.

Amestoy et al. ${ }^{26}$ realizaram um estudo com o objetivo de conhecer o entendimento de enfermeiros sobre o exercício da liderança dialógica no ambiente hospitalar, bem como os desafios enfrenta- dos para exercer a liderança. Os autores observaram que a liderança dialógica é entendida como a capacidade do enfermeiro de coordenar e de organizar a equipe de enfermagem a partir de relações horizontalizadas, norteadas pelo diálogo. Quanto aos desafios, os autores destacaram as relações hierárquicas e de poder e a falta de experiência profissional, que também foi mencionada por Ferreira et al. ${ }^{10} \mathrm{em}$ seu estudo.

No estudo de Ryder et al. ${ }^{27}$ é possível identificar a falta de confiança na pesquisa como um desafio dos enfermeiros. Os autores exploraram as maneiras pelas quais os enfermeiros-líderes implementam liderança e pesquisa em suas funções e concluíram que enfermeiros são líderes clínicos focados em melhorar a prestação de cuidados de saúde às populações de pacientes, porém, não têm confiança para realizar pesquisas de forma independente, fazendo com que a pesquisa necessite do apoio de enfermeiros da academia.

Por fim, Fast,Rankin ${ }^{28}$ levantaram a questão do uso de tecnologias como um desafio no ambiente hospitalar. Segundo os autores, orçamentos apertados e um conjunto crescente de tecnologias que direcionam as decisões em relação a quantidade suficiente de pessoal, tem moldado as responsabilidades dos gerentes aos objetivos institucionais, dificultando assim, a prática da liderança.

\section{CONCLUSÃO}

A literatura apresentou dados e informações relevantes que permitiram entender quais são os principais desafios dos enfermeiros na liderança em enfermagem. Foram identificados 12 desafios, sendo que, "desenvolver habilidades de liderança” foi o principal desafio abordado.

Sugere-se a divisão dos desafios em três grandes grupos para facilitar sua visualização, sendo eles: Desenvolver habilidades de liderança e inteligência emocional; Promover um bom ambiente de trabalho, estimulando a autonomia e o trabalho em equipe; e Superar barreiras relacionadas à questôes de gênero, falta de experiência 


\section{artigo}

profissional, falta de confiança, uso de novas tecnologias e questões políticas e hierárquicas.

Ao analisar estes grandes grupos, po- de-se notar uma certa complexidade em superar estes desafios na prática. Dessa forma, sugere-se a realização de novos estudos acerca da prática da liderança em enfermagem, com o intuito de auxiliar na superação destes desafios, apoiando assim, melhorias na prática da liderança por enfermeiros. -

\section{REFERÊNCIAS}

1.Carvalho AGF, Cunha ICKO, Balsanelli AP, Bernardes A. Liderança autêntica e perfil pessoal e profissional de enfermeiros. Acta Paulista de Enfermagem. 2016; 29(6); 618-625.

2. COFEN - Conselho Federal de Enfermagem. Lei n 7.498 , de 25 de junho de 1986. Dispõe sobre a Regulamentação do Exercício da Enfermagem e dá outras providências. Diário Oficial da União. Brasília (DF).1986. Seção I - fls. 9.273 a 9.275. Disponível em: <http://www.cofen.gov.br/lei-n-749886-de-25-de-junhode1986_4161.html>.

3. Filho JCS, Silva INN, Oliveira JAL, Barbosa MS, Azevedo JEC, Galvão MESM. 0 papel do enfermeiro na gestão de qualidade: revisão de literatura. Revista Saúde Coletiva. 2019; (09) N.48.

4.Gil AC. Como elaborar projetos de pesquisa. 5 a ed. São Paulo: Atlas; 2010.

5.Wardani E, Ryan T. Barriers to nurse leadership in an Indonesian hospital setting. J Nurs Manag. 2019; 27(3):671-678.

6.Correa PB, Bacon CT. The Effect of Leadership Interventions on Staff Nurse Job Enjoyment and Leadership Perception. The journal of nursing administration. 2019; Vol. 49; No. 4.

7.Anderson C. Exploring the role of advanced nurse practitioners in leadership. Nursing Standard. 2018; Vol 33; No 2.

8.Major D. Developing effective nurse leadership skills. Nursing Standard. 2019; Vol 34; № 6.

9.Pereira MV, Spiri WC, Spagnuolo RS, Juliani CMC. Transformational leadership: journal club for emergency and intensive care nurse managers. Revista Brasileira de Enfermagem. 2020; 73(3); e20180504.

10.Ferreira VB, Amestoy SC, Silva GTR, Trindade LL, Santos IAR, Varanda PAG. Transformational leadership in nursing practice: challenges and strategies. Revista Brasileira de Enfermagem. 2020; 73(6); e20190364.

11.Dyess S, Sherman R, Pratt B, Chiang-Hanisko L. "Growing Nurse Leaders: Their Perspectives on Nursing Leadership and Today's Practice Environment" OJIN: The Online Journal of Issues in Nursing. 2016; Vol. 21; No. 1.

12. Henriques Camelo SH, Rossi Rocha FL, Días Pedreschi Chaves L, Santos Silva VL, Inácio Soares M. Competências profissionais e estratégias organizacionais de gerentes de enfermagem. Ciencia y enfermería. 2016; 22(1); 75-86.

13.Van der Heijden BI, Mulder RH, König C, Anselmann V. Toward a mediation model for nurses' well-being and psychological distress effects of quality of leadership and social support at work. Medicine. 2017; 96(15):e6505.

14.Furunes T, Kaltveit A, Akerjordet K. Health promoting leadership: A qualitative study from experienced nurses' perspective. J
Clin Nurs. 2018; 27:4290-4301.

15.Raso R, Fitzpatrick JJ, Masick K. Clinical Nurses' Perceptions of Authentic Nurse Leadership and Healthy Work Environment. The journal of nursing administration. 2020; Vol. 50; No. 9.

16. Poghosyan L, Liu J. Nurse Practitioner Autonomy and Relationships with Leadership Affect Teamwork in Primary Care Practices: a Cross-Sectional Survey. J Gen Intern Med. 2016; 31; 771-777.

17. Silva LJ, Leite JL, Silva TP, Silva I R, Mourão PP, Gomes TM, Management challenges for best practices of the Kangaroo Method in the Neonatal ICU. Revista Brasileira de Enfermagem. 2018; 71(Suppl. 6); 2783-2791.

18. Richter SA, Santos EP, Kaiser DE, Capellari C, Ferreira GE. Ações empreendedoras em enfermagem: desafios de enfermeiras em posição estratégica de liderança. Acta Paulista de Enfermagem. 2019; 32(1); 46-52.

19.Oliveira C, Santos LC, Andrade J, Domingos TS, Spiri WC. Leadership in the perspective of Family Health Strategy nurses. Revista Gaúcha de Enfermagem. 2020.

20.Prezerakos PE. Nurse Managers' Emotional Intelligence and Effective Leadership: A Review of the Current Evidence. The open nursing journal. 2018; 12; 86-92.

21.Al-Motlaq M. Nurse Managers' Emotional Intelligence and Effective Leadership: A Review of Current Evidence. The open nursing journal. 2018; 12; 225-227.

22.Mansel B, Einion A. 'It's the relationship you develop with them': emotional intelligence in nurse leadership. A qualitative study. British Journal of Nursing. 2019; Vol 28; No 21.

23.Frasier N. Preparing Nurse Managers for Authentic Leadership. The journal of nursing administration. 2019; Vol. 49; No. 2.

24.Ngabonzima A, Asingizwe D, Kouveliotis K. Influence of nurse and midwife managerial leadership styles on job satisfaction, intention to stay, and services provision in selected hospitals of Rwanda. BMC nursing. 2020; 19; 35.

25.Balsanelli AP. Liderança em enfermagem: Desafios e possibilidades. Acta Paulista de Enfermagem. 2017; 30(1); 3-4.

26.Amestoy SC, Oliveira AFL, Thofehrn MB, Trindade LL, Santos BPD, Bao ACP. Contributions of Paulo Freire to understanding the dialogic leadership exercise of nurses in the hospital setting. Rev Gaucha Enferm. 2017; 38(1):e64764.

27.Ryder $M$, Jacob $E$, Hendricks J. An inductive qualitative approach to explore Nurse Practitioners views on leadership and research: An international perspective. J Clin Nurs. 2019; 28:2644-2658.

28.Fast 0, Rankin J. Rationing nurses: Realities, practicalities, and nursing leadership theories. Nurs Inq. 2018; 25:e12227. 